\title{
Flow Diversion in Ruptured Blood Blister Aneurysms: Single Centre Experience
}

\author{
Dilip Kumar ${ }^{1}$ Rahul K.R. ${ }^{1} \quad$ Santhosh P. ${ }^{1} \quad$ Pankaj Mehta $^{1} \quad$ Mathew Cherian $^{1} \quad$ Dharav Kheradia $^{2}$ \\ Rinoy R. Anand ${ }^{3}$
}
${ }^{1}$ Division of Neuro and Vascular Interventional Radiology, Department of Radiology, Kovai Medical Centre and Hospital, Coimbatore, Tamil Nadu, India
${ }^{2}$ Department of Interventional Radiology, Baroda, Gujarat, India
${ }^{3}$ Department of Radiology, Kovai Medical Centre and Hospital,
Coimbatore, Tamil Nadu, India

J Clin Interv Radiol ISVIR 2017;1:77-84.

\begin{abstract}
Keywords

- blister aneurysm

- flow diverter

- subarachnoid hemorrhage

Blood blister aneurysms are extremely rare, and its treatment continues to be controversial. Although surgery was the only available treatment a decade ago, today newer endovascular devices such as flow diverters (FDs) appears to have very low complication rates with good long-term results. We analyzed our data of seven patients who angiographically had features of a blister aneurysm. All these patients presented with subarachnoid hemorrhage $(\mathrm{SAH})$. Six of these were in anterior circulation, all of which were in internal carotid artery (ICA) and one was in a P1 segment of posterior cerebral artery (PCA). All of the patients except one in PCA were treated with FDs. One of the patients died (14.3\%) following the procedure secondary to thrombosis of FD. Rest of the patients did well postprocedure with the good clinical outcome (modified Rankin Scale [mRS] of less than or equal to 2 at 1 month follow-up) in $85.7 \%$ patients. Five of the patients showed complete obliteration of an aneurysm (83.3\%) on 6-month follow-up angiography. The only patient with an aneurysm in PCA showed persistence of an aneurysm and this particular lesion was instead treated by a single stent. Blister aneurysms pose diagnostic and therapeutic challenges and demand prompt treatment. Considering that all patients who were treated with FD had complete obliteration, it can be inferred that FD can be the treatment of choice in patients with blister aneurysms presenting with SAH. Further, with the introduction of small vessel FD, thrombotic complications may reduce, lowering the morbidity and mortality. Isolated stenting may not be an optimal treatment of a blister aneurysm.
\end{abstract}

\section{Introduction}

Blister aneurysms are the small, broad-based, hemispherical protrusions arising at nonbranching sites of intradural internal carotid artery (ICA). ${ }^{1-3}$ These lesions are rare, constituting 0.5 to $2 \%$ of ruptured aneurysms and have high morbidity and mortality compared with ruptured saccular aneurysms. Blister aneurysms are a specific type of dissection/pseudoa- neurysm which demands treatment of the affected wall of ICA along with the blister rather than focusing on treatment of the aneurysm sac alone. ${ }^{4}$ Numerous surgical and endovascular procedures have been described in the management of this distinct entity. Recently, flow diverters (FDs) have been employed in treating such complex, difficult to treat blister aneurysms. ${ }^{5}$ We share our experience in the 
treatment of ruptured blood blister aneurysms in seven patients emphasizing the importance of flow diversion technique.

\section{Material and Methods}

We reviewed the data of all cerebral aneurysms that were treated at our center between 2014 and 2016. From the data, we retrospectively analyzed seven patients of blister aneurysms who presented with subarachnoid hemorrhage. We included those patients with ruptured blister-like aneurysms at the nonbranching sites of intracranial circulation. Patients with aneurysms adjacent to posterior communicating, anterior choroidal, and superior hypophyseal artery and aneurysms of saccular configuration were excluded from the study. All patients were treated with parent artery reconstruction using FDs except one patient with P1 segment posterior cerebral artery (PCA) blister who was treated with single stenting. Clinical presentation, diagnostic angiogram, procedural data, postprocedural clinical and angiographic follow-up data were retrospectively analyzed.

All the patients were admitted to the intensive care unit after getting a plain computed tomography brain study. A diagnostic cerebral angiogram was performed after thorough clinical examination by neurosurgeon, neurointerventionist, and neurologist. All studies were done in biplane digital subtraction angiography suite (Siemens Artis) with the facility of three-dimensional (3D) rotational angiography. Following diagnostic angiogram, further management plans regarding parent artery reconstruction, risks associated with procedure including the radiation hazards were discussed with patient relatives and consent was obtained for the same. All patients received a loading dose of tablet aspirin $300 \mathrm{mg}$ ( 3 hours before the procedure) and a loading dose of tab ticagrelor $180 \mathrm{mg}$ on the table during intubation via a nasogastric tube. The procedure was done under general anesthesia. A 7F long sheath was placed in the distal common carotid artery via right common femoral artery access. 3D spin was acquired and after generating the working angle $6 \mathrm{~F}$ neuron-guiding catheter was placed in cavernous ICA. Under roadmap guidance, the microcatheter with Traxcess EX guide wire (Microvention Inc.) combination was navigated across the aneurysm. Marksman microcatheter and headway 27 microcatheter were used to deploy pipeline embolization device (PED) and flow redirection endoluminal device (FRED, Microvention, Terumo, United States), respectively. Surpass FD comes preloaded on a delivery microcatheter deployed via distal access catheter. Flow diverter stents (PED [Medtronic], FRED [Microvention], and SURPASS [Stryker]) were used in all ICA blister aneurysms, and simple stent (lowprofile visualized intraluminal support device [LVIS], Microvention) was used in P1 segment PCA blister aneurysm. Target activated clotting time values were maintained between 250 and 300 seconds during the procedure. Intraarterial tirofiban was given if the features of stent thrombosis were seen on a postprocedure angiogram.

Patients were discharged with dual antiplatelet (comprising of tablet aspirin $75 \mathrm{mg}$ once daily and tablet ticagrelor $90 \mathrm{mg}$ twice daily for 6 months) clinically followed up in outpatient department at 1 and 6 months. Check cerebral angiogram was done at 6 months following which patient was put on tab aspirin $75 \mathrm{mg}$ once daily for a lifetime.

\section{Results}

Summary of patient details comprising age, sex, aneurysmal site, complications, clinical follow-up with mRS at 30 days and angiographic follow-up at 6 months are presented in -Table 1. Six patients were males, and one was female with the mean age of 55.5 years (range: $37-70$ years). All of them presented with SAH, caused by rupture of blister-like an aneurysm having a Fisher grade 3 . All patients had a good Glasgow Coma Scale. ${ }^{6,7}$ No patients had hydrocephalus requiring an external ventricular drain. Anatomical location of blisters were two in supraclinoid ICA segment, four in paraclinoid ICA segment, and one in a P1 segment of the posterior cerebral artery. No patients had cardiac ailments or smoking history, two had hypertension, and two patients had diabetes mellitus.

Out of seven patients, six had a frank blister-like aneurysm at first diagnostic angiogram. One of the patients had a doubtful lesion and posed a diagnostic challenge in further management (-Fig. 1). However, magnetic resonance imaging vessel wall imaging demonstrated intramural hematoma and check cerebral angiogram 1 week later revealed that suspected aneurysm had increased in size confirming blister pathology.

All ICA blister patients were treated with parent artery reconstruction using FD stents. Two PEDs, three FREDs, and one SURPASS FDs were used. Distal landing zone of FDs was M1 middle cerebral artery (MCA) in three patients, communicating ICA segment in three patients. One patient with PCA a blister aneurysm was treated with single LVIS stent. None of the aneurysms were coiled. Four out of six ICA blister patients showed contrast stasis in an aneurysm on a postprocedural angiogram (-Fig. 2). One of the ICA blister patients had small thrombus formation soon after stent placement (distal landing of the FD was into the M1 MCA) which was managed with an $11 \mathrm{~mL}$ bolus of intra-arterial tirofiban injection (-Fig. 2).

Immediate stasis of contrast in aneurysmal sac following FD deployment was observed in four out of six patients (66\%). One ICA blister patient died (mortality, 14.3\%) on fourth postoperative day secondary to thrombosis of FD stent and malignant MCA infarct. In this patient, the distal landing of FD was into the M1 MCA. Rest of the patients did well postprocedurely with $\mathrm{mRS}$ of less than or equal to 2 at 1-month follow-up, accounting for good clinical outcome in $85.7 \%$ patients. All of the six living patients had follow-up angiogram at 6 months. Five of the ICA blister patients showed complete obliteration of the aneurysm. Thus $83.3 \%$ of ICA blister patients treated with FD had complete obliteration of an aneurysm. The only patient with an aneurysm in PCA showed the persistence of an aneurysm and this particular lesion was not treated by FD, 


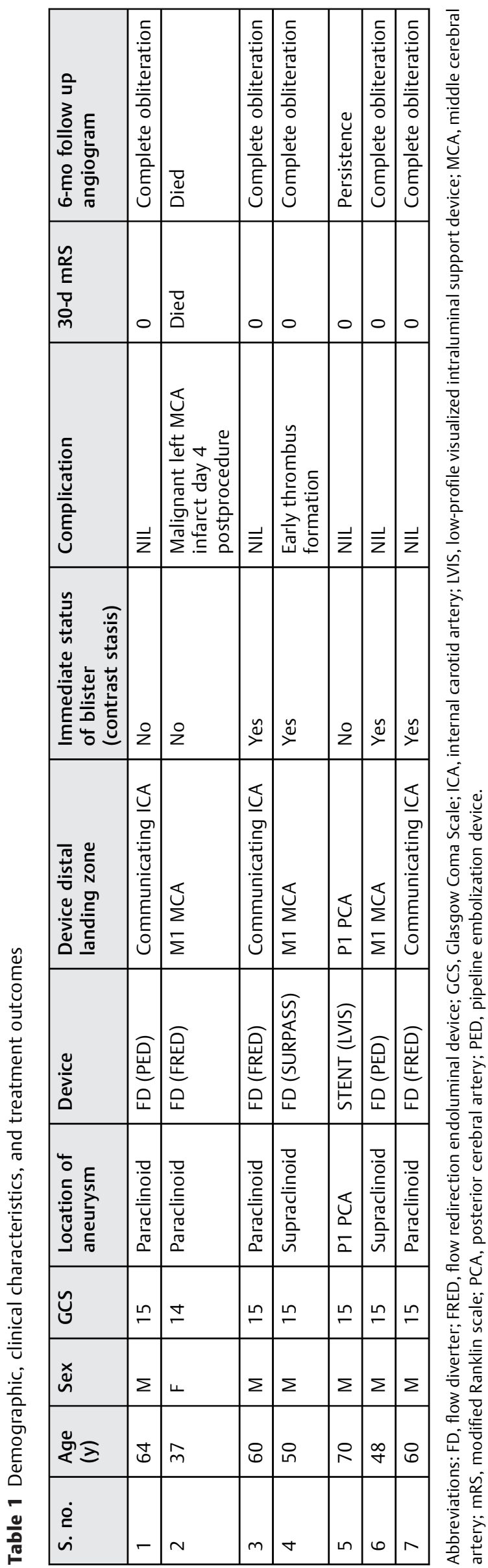

instead by a single stent (-Fig. 3). This particular patient was advised FD placement at the earliest and is on regular follow-up.

\section{Discussion}

Classically, the blister aneurysms are small broad-based hemispherical protrusions arising at nonbranching sites of intradural ICA. ${ }^{1-3}$ Nakagawa et al first described this distinct entity in 1986, and Takahashi et al coined the term blister in 1988. The typical location is supraclinoid ICA; however, anterior communicating artery, vertebra-basilar circulation can also be involved. These lesions are rare, constituting 0.5 to $2 \%$ of ruptured aneurysms and have high morbidity and mortality compared with ruptured saccular aneurysms. ${ }^{4}$ Preprocedure diagnostic angiogram may miss the diagnosis of blister because of their smaller size. However, because of rapid growth, the sensitivity of repeat catheter angiogram increases significantly. ${ }^{8}$ In contrast to the wall of a berry aneurysm which consists of collagen within thickened intima and adventitia, a blister is a focal defect in the intima and media covered by a thin adventitia. ${ }^{2,9}$ Thus blister aneurysms are a specific type of dissection/pseudoaneurysm which demands treatment of the affected wall of ICA along with the blister rather than focusing on treatment of the aneurysm sac alone. ${ }^{4}$

Different surgical procedures have been reported including clipping, wrapping, trapping, trapping with clipping and bypass, trapping with balloon and bypass, primary bypass, and arterial suturing. ${ }^{9}$ Endovascular procedures included coiling, balloon/stent-assisted coiling, single stent alone, multiple stents, parent artery occlusion, FD alone or with coils. $^{10}$

Because of the fragile paper-thin walls of blister aneurysms, surgical clipping met with high rate of intraoperative ruptures which finally led to sacrificing the parent artery. ${ }^{8,11-13}$ For the same reason, primary or stent-assisted coiling of blisters carry a high risk of rupture and hemorrhage. $6,14,15$ Parent artery occlusion by surgical or endovascular means may be the most definitive treatment of blister aneurysms following compliant balloon test. However, Meling et al reported poor outcome following parent artery occlusion despite good preoperative collateral capacity. ${ }^{7}$ They hypothesized that vasospasm would reduce the collateral capacity leading to delayed ipsilateral cerebral ischemia after parent artery occlusion.

Antiplatelet medications are the issue while considering endovascular treatment using stent/FDs as they expose the patient to high risk of rebleed following treatment and makes external ventricular drain unsuitable if in case the patient develops hydrocephalus in the postoperative period.

Numerous studies have evaluated the outcomes with endovascular treatment. The largest meta-analysis of treatment and outcomes in blister aneurysms till date by Peschillo et al showed that endovascular treatment had lower morbidity and mortality, and better outcomes to surgical approaches. ${ }^{10}$ Stenting alters flow dynamics within the 

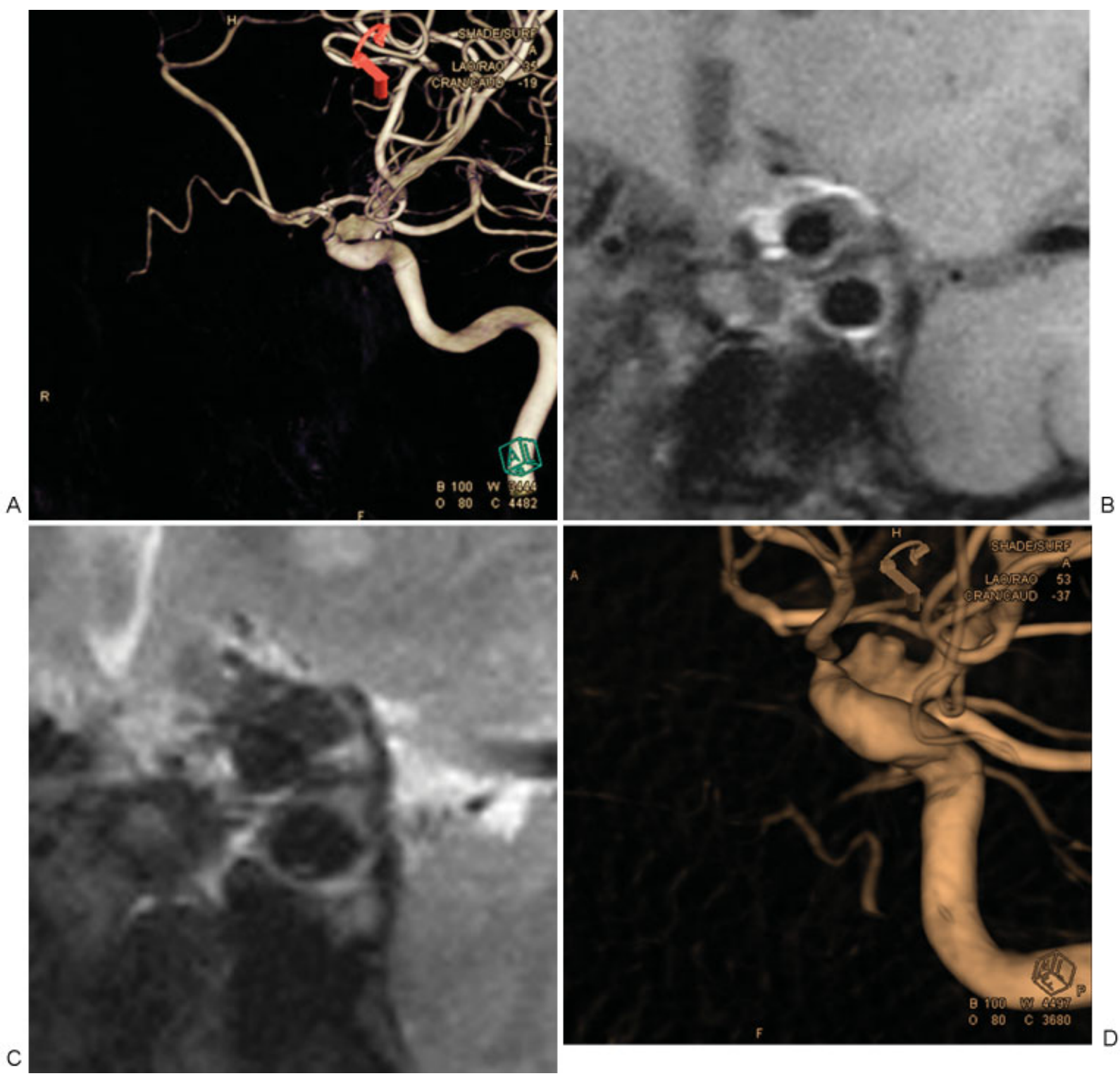

Fig. 1 (A) 3D spin (3D rotational angiogram) shows doubtful supraclinoid ICA blister lesion. (B) Reformatted cross-section T1 MRI vessel wall imaging of the ICA shows crescentic hyperintensity in the wall of supraclinoid ICA suggestive of Intramural hematoma. (C) Reformatted crosssection T2 MRI vessel wall imaging of the ICA shows crescentic hypointensity in the wall of supraclinoid ICA suggestive of Intramural hematoma. (D) Check angiogram (3D spin) 1 week later shows rapidly enlarging blister aneurysm. (E and F) Immediate postflow diverter deployment angiogram shows filling of the aneurysm. ( $G$ and $H$ ) The 6-month follow-up angiogram shows complete obliteration of the aneurysm. 3D, threedimensional; ICA, internal carotid artery; MRI, magnetic resonance imaging.

aneurysm and provides scaffold for intimal growth reducing the likelihood of recurrence. ${ }^{16}$ Kim et al reported stentassisted coiling followed by stent within stent placement later prevents regrowth in blister aneurysms. ${ }^{17}$

Recently, FDs are employed in treating such complex, difficult to treat blister aneurysms. ${ }^{5}$ Flow diversion technique is based on the endoluminal reconstruction of parent artery and aneurysmal neck altering inflow and outflow jets subsequently leading to aneurysmal thrombosis. Over time, neointimal hyperplasia covers FD stent matrix thus remodeling the parent artery and eliminates aneurysm/ parent vessel interface. ${ }^{18,19}$
In our study, we report clinical and angiographic results for the treatment of ruptured blister aneurysms using parent artery reconstruction by FDs and single stent in a cohort of seven patients. Immediate stagnation of contrast was seen in $66 \%$ of patients following FD which is a good early sign of optimal flow diversion and may predict rapid aneurysmal obliteration in near future.

A meta-analysis done by Peschillo et al reported thromboembolic complications in $33.3 \%$ of patients in the endovascular group. This complication was much more frequent with $\mathrm{FD}$ (50\% of cases). ${ }^{10}$ In our study, two of six $(33.3 \%)$ patients treated with FD had thrombotic 

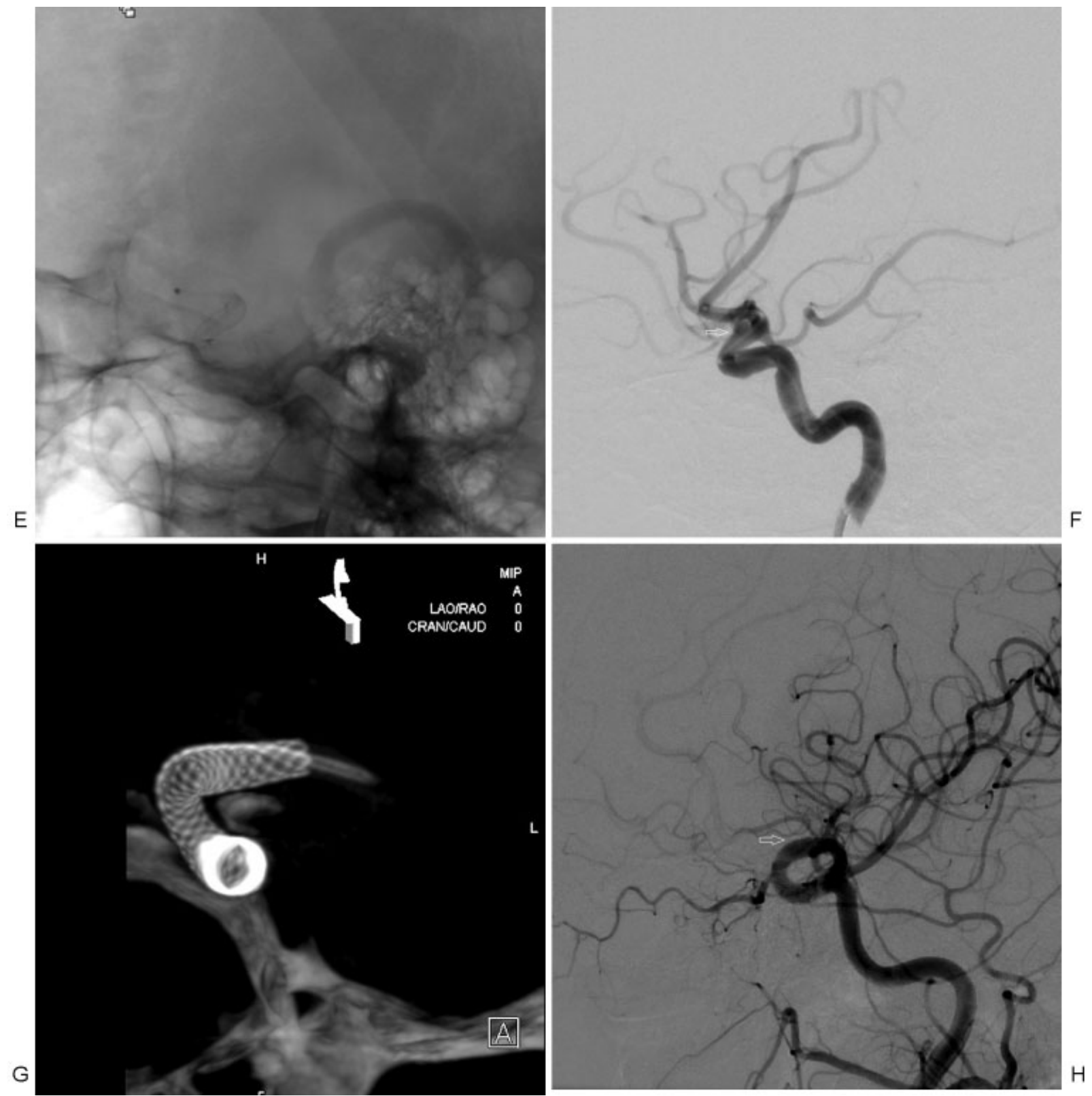

Fig. 1 Continued

complications. One patient had malignant MCA infarct due to FD thrombosis, and the other had a small thrombus formation in the stent seen on the immediate postprocedure angiogram which was contained with intra-arterial tirofiban. Chances of thrombosis of FD were higher (two out of three patients, 66.6\%) when the distal landing of the FD stent was in the M1 segment of the MCA. Thrombotic complications were not seen when the FD was limited to the communicating ICA segment. We hypothesize that size discrepancy between the proximal and distal reconstructed vessels was responsible for the thrombotic complication since a small vessel will have a greater metal density compared with the larger diameter vessel segment that is appropriate for the size of the FD. We infer that limiting the FD to a vessel with more or less uniform diameter along the entire length can dramatically reduce this dreaded complication. Also, the introduction of FDs with less metal density may lower this complication rate drastically. Suboptimal platelet suppression could be another reason for thrombotic complications since all the patients were taken up as emergency cases where only a loading dose of antiplatelet was given prior and during the procedure.

Retrospective design, small sample size, no control group, and limited knowledge on the long-term behavior of the FD stent mark the limitations of our study. 

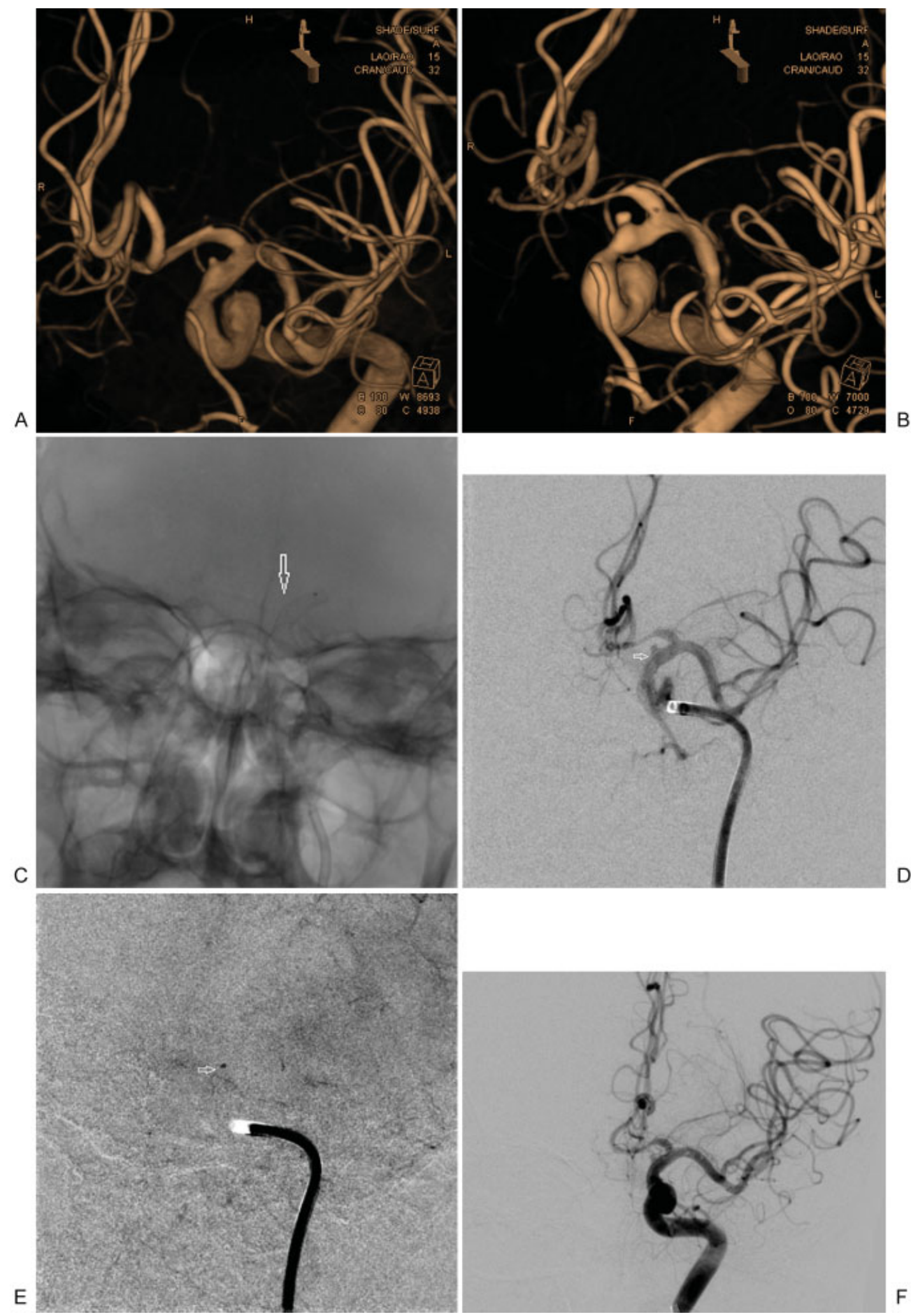

Fig. 2 (A) 3D spin angiogram shows left supraclinoid ICA blister aneurysm. (B) 3D spin angiogram 1 week later shows rapidly enlarging blister aneurysm. (C) Flow diverter in the left ICA extending into an M1 segment of left MCA. (D) Immediate postflow diverter deployment angiogram shows an aneurysm filling in arterial phase. (E) Immediate postflow diverter deployment angiogram shows stasis of contrast in an aneurysmal sac in capillary phase. (F) Postflow diverter deployment angiogram shows filling defects in M1 MCA suggestive of thrombus formation which was contained with intra-arterial tirofiban. 3D, three-dimensional; ICA, internal carotid artery; MCA, middle cerebral artery. 

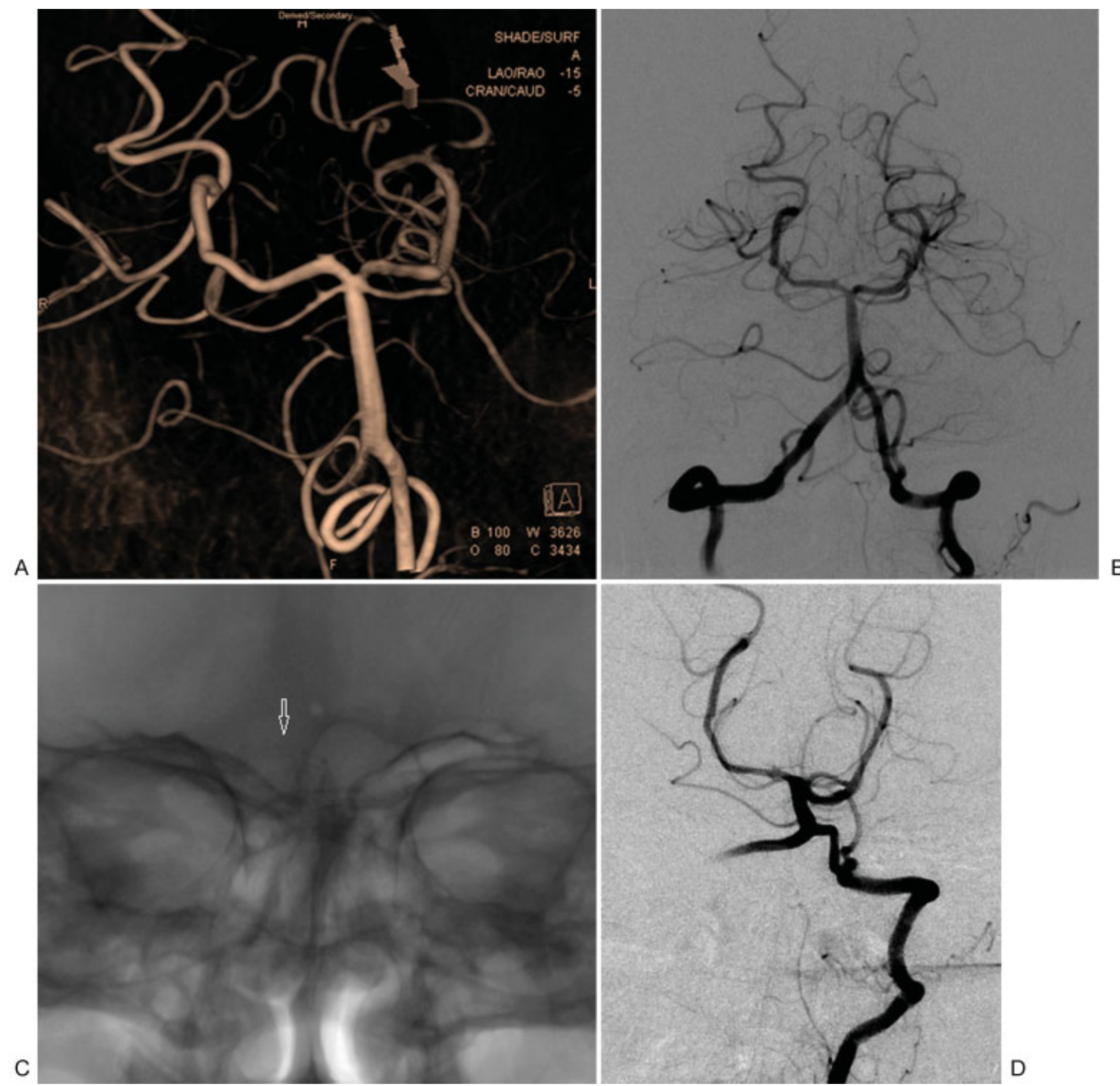

Fig. 3 (A) 3D spin shows blister aneurysm of a P1 segment of right posterior cerebral artery. (B) Immediate poststent deployment (LVIS stent, Microvention, Terumo, United States) angiogram shows aneurysm filling. (C) LVIS stent in a P1 segment of the right posterior cerebral artery. (D) A 6-month follow-up angiogram shows the persistence of the right P1 blister aneurysm. LVIS, low-profile visualized intraluminal support.

\section{Conclusion}

Blister aneurysms pose diagnostic and therapeutic challenges and demand prompt treatment. Considering that all patients who were treated with FD had complete obliteration, it may be inferred that FD can be a promising treatment option in patients with blister aneurysms presenting with SAH. However thrombotic complications pose a significant challenge in patients treated with FD. Isolated stenting may not be an optimal treatment for a blister aneurysm.

\section{References}

1 Nakagawa F, Kobayashi S, Takemae T, Sugita K. Aneurysms protruding from the dorsal wall of the internal carotid artery. J Neurosurg 1986;65(03):303-308
2 Tekkök IH, Bakar B. Ruptured blister-like aneurysm of distal internal carotid artery: a distinct entity. Turk Neurosurg 2008; 18(04):439-445

3 Takahashi A, Suzuki J, Fujiwara S, Yoshimoto T. Surgical treatment of chimame (blood blister) like aneurysm at C2 portion of internal carotid artery. Surg Cerebr Stroke 1988;16(01): $72-77$

4 Lee BH, Kim BM, Park MS, et al. Reconstructive endovascular treatment of ruptured blood blister-like aneurysms of the internal carotid artery. J Neurosurg 2009;110(03):431-436

5 Aydin K, Arat A, Sencer S, et al. Treatment of ruptured blood blister-like aneurysms with flow diverter SILK stents. J Neurointerv Surg 2015;7(03):202-209

6 Nguyen TN, Raymond J, Guilbert F, et al. Association of endovascular therapy of very small ruptured aneurysms with higher rates of procedure-related rupture. J Neurosurg 2008;108(06): 1088-1092

7 Meling TR, Sorteberg A, Bakke SJ, Slettebø H, Hernesniemi J, Sorteberg W. Blood blister-like aneurysms of the internal carotid 
artery trunk causing subarachnoid hemorrhage: treatment and outcome. J Neurosurg 2008;108(04):662-671

8 Ogawa A, Suzuki M, Ogasawara K. Aneurysms at nonbranching sites in the surpaclinoid portion of the internal carotid artery: internal carotid artery trunk aneurysms. Neurosurgery 2000; 47(03):578-583, discussion 583-586

9 Gonzalez AM, Narata AP, Yilmaz H, et al. Blood blister-like aneurysms: single center experience and systematic literature review. Eur J Radiol 2014;83(01):197-205

10 Peschillo S, Cannizzaro D, Caporlingua A, Missori P. A systematic review and meta-analysis of treatment and outcome of blisterlike aneurysms. AJNR Am J Neuroradiol 2016;37(05):856-861

11 Abe M, Tabuchi K, Yokoyama H, Uchino A. Blood blisterlike aneurysms of the internal carotid artery. J Neurosurg 1998; 89(03):419-424

12 Sim SY, Shin YS, Cho KG, et al. Blood blister-like aneurysms at nonbranching sites of the internal carotid artery. J Neurosurg 2006;105(03):400-405

13 Lee J-W, Choi H-G, Jung J-Y, Huh S-K, Lee K-C. Surgical strategies for ruptured blister-like aneurysms arising from the internal carotid artery: a clinical analysis of 18 consecutive patients. Acta Neurochir (Wien) 2009;151(02):125-130
14 Joo S-P, Kim T-S, Moon K-S, et al. Arterial suturing followed by clip reinforcement with circumferential wrapping for blister-like aneurysms of the internal carotid artery. Surg Neurol 2006; 66(04):424-428, discussion 428-429

15 van Rooij WJ, Keeren GJ, Peluso JPP, Sluzewski M. Clinical and angiographic results of coiling of 196 very small $(<$ or $=3 \mathrm{~mm}$ ) intracranial aneurysms. AJNR Am J Neuroradiol 2009;30(04): 835-839

16 Tanemura H, Ishida F, Miura Y, et al. Changes in hemodynamics after placing intracranial stents. Neurol Med Chir (Tokyo) 2013; 53(03):171-178

17 Kim BM, Chung EC, Park SI, Choi CS, Won YS. Treatment of blood blister-like aneurysm of the internal carotid artery with stent-assisted coil embolization followed by stent-within-astent technique. Case report. J Neurosurg 2007;107(06): 1211-1213

18 Zanaty M, Chalouhi N, Tjoumakaris SI, Rosenwasser RH, Gonzalez LF, Jabbour P. Flow-diversion panacea or poison? Front Neurol 2014;5:21

19 Alderazi YJ, Shastri D, Kass-Hout T, Prestigiacomo CJ, Gandhi CD. Flow diverters for intracranial aneurysms. Stroke Res Treat 2014; 2014(14):415653 